\title{
Finite element modeling of actuated fibre composites
}

\author{
M. Martinez ${ }^{1}$, A. Artemev², F. Nitzsche ${ }^{2}$ \& B. Geddes ${ }^{2}$ \\ ${ }^{I}$ National Research Council of Canada (NRC), \\ Institute for Aerospace Research, Ottawa, Ontario, Canada \\ ${ }^{2}$ Department of Mechanical and Aerospace Engineering, \\ Carleton University, Ottawa, Ontario, Canada
}

\begin{abstract}
A finite element method (FEM) study of the actuation and sensing performance of actuated fibre composites (AFC) is presented. The effect of non-continuous fibres on the AFC performance was analyzed for actuation and sensing applications. The results of the FEM analysis of AFC with non-continuous fibres are compared to experimental results obtained in specimens with fibres damaged by large deformation. A study of the change in the polarization state in the fibre, resulting from the formation of a gap, was performed and its results were incorporated into the FEM. The correlation between the available experimental data and simulation results is discussed.
\end{abstract}

Keywords: PZTs, actuated fibre composites, AFCs, piezoelectric fibres, sensors, smart rotor.

\section{Introduction}

Modern military and civilian aircraft structures require the use of monitoring systems to identify and predict the health of critical components within the aircraft. Health Prognostic Monitoring (HPM) is in the process of being introduced to modern aircraft structures. This concept will allow the aircraft to determine if the life of a critical component is coming to an end, and have the overall system schedule the time and place for the component to be repaired/replaced. Sensors are required to acquire the current state of strain, temperature or other parameters of concern in specific areas of the aircraft, which is a critical part of HPM. Several sensors show promise for these 
applications, such as, strain gauges, fibre optic strain sensors and piezoelectric sensors. The use of piezoelectric sensors and actuators embedded in laminated composites is also being studied as a means of reducing noise and vibration generated by helicopter rotors. A "Smart Blade" structure would be capable of reacting to an external electric stimulus measured by AFC sensors and allow for the deformation of the rotor blade via AFC actuation. This paper concentrates on the use of piezoelectric fibres as strain sensors. The "Smart Blade" tailors the aerodynamic load distribution to attenuate both noise and vibration at flight conditions spanning from vertical take-off, manoeuvring flight, high-speed cruise and vertical landing.

The use of piezoelectric materials for both actuation and sensing functions has made them attractive for use in micro electro mechanical systems [1] as well as for large-scale applications. For example, piezoelectric materials are being analyzed for active control of airfoils [2-4]. However, bulk piezoelectric materials have several serious drawbacks impeding their applications. In particular, these ceramic materials have inherently low toughness. A number of brittle materials have been used, in the form of fibres, in fibre-reinforced composites. Such materials allow for the use of high strength fibres together with a high toughness developed in a composite structure. Recently, several attempts have been made to develop composite materials with piezoelectric fibres [5-7]. Such actuated fibre composites (AFC) can be used as actuators and/or sensors in a number of different applications.

The use of AFC for practical applications is still restricted by the lack of detailed knowledge about the relationship between the structure, manufacturing process, and performance of the material. Currently, for several AFC systems, research is being conducted on the relationships between structures, properties of the constituent components, and the behavior of the system as a whole [8-10]. One particularly challenging problem is the analysis of long-term behavior and degradation of AFC materials. Studies into the effect of fibre damage on the performance of AFC are an integral part of this research [11-12]. The presence of gaps within the fibre has significant consequences for the polarization field of the fibre. This change in the proximity of the gap size has a major impact on the electric potential distribution and, thus, on the overall response obtained from the AFC. This study has been carried out using a custom developed FEM solver [13].

\section{Domain patterns in broken fibres}

A phase field model of ferroelectric materials developed in [14] was used to study the effect on the polarization of gaps in fibres. In this model the distribution of the order parameter vector $\boldsymbol{\eta}$ describes the state of the system.

The polarization at any point is determined as $P=P_{0} \cdot \boldsymbol{\eta}$. The free energy is:

$$
F=\int\left[\frac{\beta}{2} \cdot \sum_{p=1}^{3}\left(\nabla \eta_{p}\right)^{2}+f(\boldsymbol{\eta})\right] d^{3} r+2 \pi P_{0}^{2} \sum_{p=1}^{3} \sum_{q=1}^{3} \int \frac{d^{3} k}{(2 \pi)^{3}} \frac{k_{p} k_{q}}{k^{2}} \tilde{\eta}_{p}(\mathbf{k}) \tilde{\eta}_{q}(\mathbf{k})^{*}
$$


where $\beta$ is the gradient energy coefficient, $\boldsymbol{\delta}$ is Kronecker delta symbol, $f(\boldsymbol{\eta})$ is the chemical energy, $P_{0}$ is the equilibrium polarization, $\tilde{\eta}$ is the Fourier transform of $\eta$, and $*$ indicates a complex conjugate. We used:

$$
f(\boldsymbol{\eta})=-0.136 \cdot \sum_{i} \eta_{i}^{2}-7.076 \cdot \sum_{i} \eta_{i}^{4}+4.352 \cdot\left(\sum_{i} \eta_{i}^{2}\right)^{2}+1.86 \cdot\left(\sum_{i} \eta_{i}^{2}\right)^{3}
$$

and other model parameters characterized by the dimensionless combinations $\lambda=P_{0}^{2} / \Delta f=10$ and $\beta /\left(\Delta f \cdot l^{2}\right)=0.5$, where $\Delta f$ is the difference between the chemical energies of paraelectric and equilibrium ferroelectric phases. The unit length is equal to the mesh cell length. A $512 \times 64 \times 64$ equilateral rectangular mesh with a fibre (diameter of 48 and length of 512 cells) was used. Fibres with different gap lengths were simulated. Initially the fibres had homogeneous longitudinal polarization (Fig.1(a)). The equilibrium domain patterns were found using the fast Fourier transform for the solution of the stochastic time-dependent Ginzburg-Landau equation [14] for the minimization of free energy.

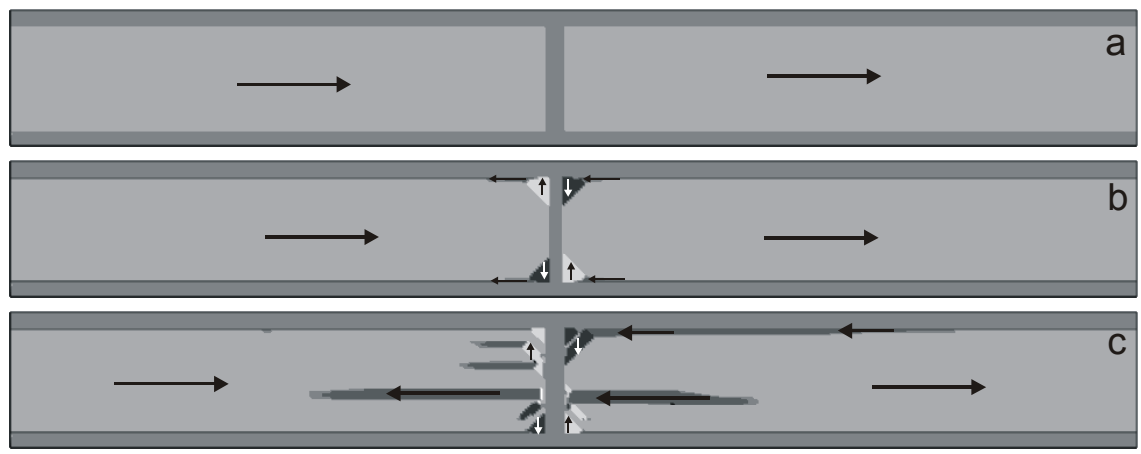

Figure 1: (a) Domain patterns in the broken fibre in the initial state and (b) after relaxation in fibres with a 6 cell long gap and (c) with a 9 cell long gap. The polarization direction is shown by arrows.

Figure 1 illustrates a longitudinal section of the system in the initial state (Fig. 1(a)) and after the completion of the simulation (Fig. 1(b) and (c)). The analysis shows that the presence of the gap results in the appearance of $90^{\circ}$ closing domains at the ends of the fibre and $180^{\circ}$ domains with reverse polarization extending along the fibre from the fibre end. The formation of this domain structure leads to a partial decrease in the overall polarization near the fibre ends. The simulation shows that increasing the gap length results in an increase in the degree of reduction of the average polarization and the increase in the length of the affected zone. Computational constraints do not allow simulation of fibres with realistic dimensions. However, the simulation results clearly indicate that the formation of a polydomain zone with a reduced poling effect can be expected near the ends of broken fibres. The depth of such a zone for real fibres can be estimated as approximately equal to the fibre diameter. 


\section{Finite element models of AFC}

Two Finite Element (FE) models were constructed. The first model, for the simulation of sensing applications, consisted of an AFC subjected to a strain of 0.9 micro strains. The second model for the simulation of actuation applications represented an AFC subjected to $900 \mathrm{~V}$. In both models the effect of gaps with and without a depolarized field was studied. The models were constructed using the material properties for PZT-5A fibres, an epoxy matrix and two Kapton ${ }^{\mathrm{TM}}$ layers. The piezoelectric fibres in the AFC model have the following piezoelectric parameters: $\mathrm{d}_{31}$ of $440 \cdot 10^{-12} \mathrm{C} / \mathrm{N}, \mathrm{d}_{33}$ of $185 \cdot 10^{-12} \mathrm{C} / \mathrm{N}$, and $\mathrm{d}_{24}$ and $\mathrm{d}_{15}$ of $560 \cdot 10^{-12} \mathrm{C} / \mathrm{N}$. Mechanical properties were represented by $\mathrm{C}_{11}=120 \mathrm{GPa}$, $\mathrm{C}_{33}=110 \mathrm{GPa} \mathrm{C}_{44}=21 \mathrm{GPa}, \mathrm{C}_{66}=23 \mathrm{GPa}, \mathrm{C}_{12}=75.2 \mathrm{GPa}$ and $\mathrm{C}_{13}=75.1 \mathrm{GPa}$ [13]. The epoxy had a Young's modulus of $2.58 \mathrm{GPa}$ and Poisson's ratio of 0.38 . The Kapton $^{\mathrm{TM}}$ had a Young's modulus of $2.5 \mathrm{GPa}$ and Poisson's ratio of 0.34 .

\subsection{Sensing AFC Model}

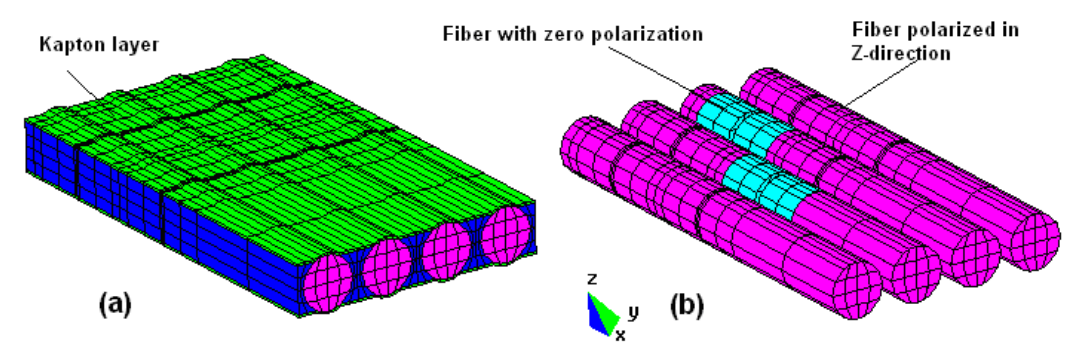

Figure 2: (a) The AFC model used for simulation of the sensing application shown with an epoxy matrix, (b) inner components of the AFC model.

The sensing AFC system was set to $2100 \mu \mathrm{m}$ in length, $1200 \mu \mathrm{m}$ in width and $260 \mu \mathrm{m}$ in thickness. It consisted of four piezoelectric fibres of $254 \mu \mathrm{m}$ diameter and an epoxy matrix contained between two Kapton ${ }^{\mathrm{TM}}$ layers, as shown in Fig. 2(a). Figure 2(b) shows the same model with the Kapton ${ }^{\mathrm{TM}}$ removed and a transparent epoxy matrix. An assumption was made that due to the presence of a $30 \mu \mathrm{m}$ gap, the regions of the fibre next to the gap with the length equal to the fibre diameter, had a zero poling effect. This means that piezoelectric constants in these regions were equal to zero; however, elasticity constants were the same as in the rest of the fibre. A defined strain of 0.9 micro strains was applied in an extension mode. The electric potential difference was measured between the top and bottom of the fibres.

\subsection{Actuation AFC model}

The unconstrained actuation AFC system was set to $4600 \mu \mathrm{m}$ in length, $1200 \mu \mathrm{m}$ in width, and $260 \mu \mathrm{m}$ in thickness. It consisted of four piezoelectric fibres, an 
epoxy matrix, and copper inter-digitized electrodes (IDE) that were covered by a thin layer of epoxy. Figure 3(a) shows a view of the AFC model including the epoxy matrix. Figure 3(b) shows the same AFC with the epoxy matrix made transparent to show the fibres and the copper electrodes. PZT fibres with $250 \mu \mathrm{m}$ diameters were spaced $300 \mu \mathrm{m}$ center to center. The AFC was cantilevered at one end. The fibres had a varying polarization field along their length. The fibre under the electrodes was modeled to have a zero polarization.

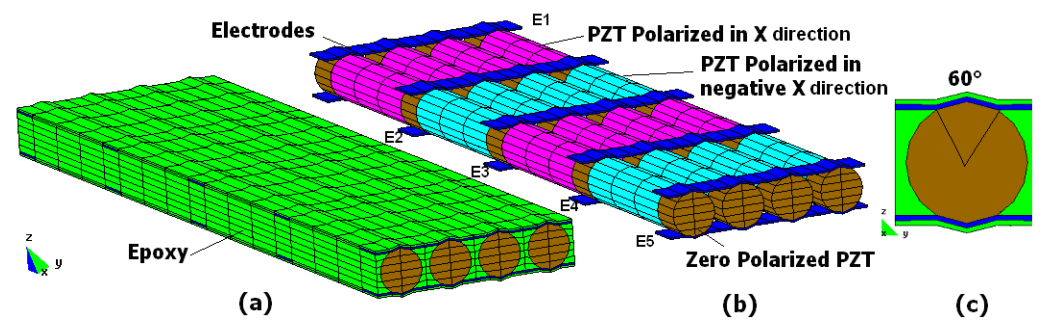

Figure 3: (a) AFC with epoxy matrix, (b) inner components of AFC, (c) cross-sectional view of AFC at the electrodes showing a $60^{\circ}$ contact angle between the electrode and the PZT fibre.

The fibres between the electrodes have a polarization along either positive or negative x-direction, as shown in Fig. 3(b). Switching of the polarization direction permits the AFC to extend or contract, since the direction of the electric field also changes from one electrode pair to another. This type of AFC structure and actuation method were presented and studied in [5-6]. The four copper electrodes are spaced $900 \mu \mathrm{m}$ apart and are $200 \mu \mathrm{m}$ wide and $10 \mu \mathrm{m}$ thick. The electrodes in Fig. 3(b) are labeled E1, E2, E3 and E4. The electric potentials for E2 and E4 are set to zero, while E1 and E3 are set to electric potentials of 900, 1350, and 1800 volts for different simulation runs. The copper electrodes make direct contact with the fibres through an angle of $60^{\circ}$, as shown in Fig. 3(c). In order to study the effect of broken fibres on the actuation performance of the AFC, we created models with different numbers of gaps $(0,1,2,5,10$ and 20) introduced into fibres. We used models in which such gaps were introduced without changing the polarization state in the rest of the fibre volume, as well as models in which gaps were accompanied by depolarized regions at the ends of the fibres with a length equal to the fibre diameter.

\section{Results of FEM analysis}

\subsection{Results of sensing application analysis}

Figure 4 demonstrates the electric potential distribution in the AFC under the applied mechanical strain. The results obtained by the simulation of the sensing application of the AFC indicate that the change in the electric potential between 
the upper and lower surface of the system in the proximity of the gap drastically differs from the voltages developed in other regions of the AFC.
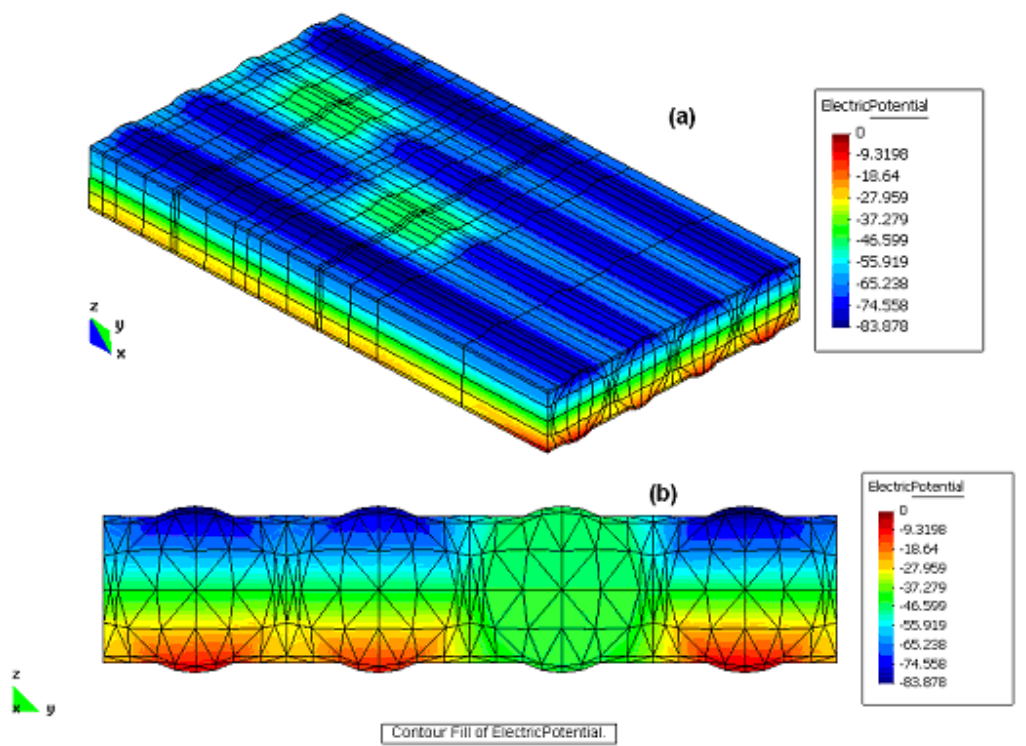

Figure 4: (a) AFC under an applied strain, (b) cross-section of AFC through the gap in the fibre.

As seen in Fig. 4, the electric potential difference through the thickness of the fibre is close to zero in the gap and in the depolarized region near it. Such loss of voltage can be a problem since discontinuities in the AFC tend to appear in the proximity of the electrodes used to apply voltage to fibres [15]. If the same electrodes were used for sensing, then they would detect a voltage corresponding to a strain that is significantly lower than the actual strain. In such a case a dual electrode systems can improve sensing performance. The first electrode group may be used for poling of the piezoelectric fibres, while the second electrode system would be used to monitor voltages produced by the piezoelectric fibres. It should be noted that in the AFC model where gaps in the fibres were not accompanied by depolarized regions, the width of the dead zone in the voltage distribution was much smaller (approximately equal to the gap width) and the loss of voltage less severe.

\subsection{Results of actuation application analysis}

The FEM analysis of the AFC under applied voltage (Fig. 5) showed that gaps in fibres can reduce the actuation performance. If a fibre between two IDE's is broken, then the electrical field is concentrated in the gap, and two pieces of fibre between electrodes have a very low electrical field applied, producing no contribution to the actuation. However, any significant reduction in the overall 
actuation performance of the AFC is obtained only when a large number of gaps is introduced effectively creating a short fibre composite. It is unlikely that such a density of damage can be developed before overall failure. The FEM results also indicate that there is very little difference between systems with depolarization regions added to the gaps in fibres and systems with gaps only (without depolarization regions).
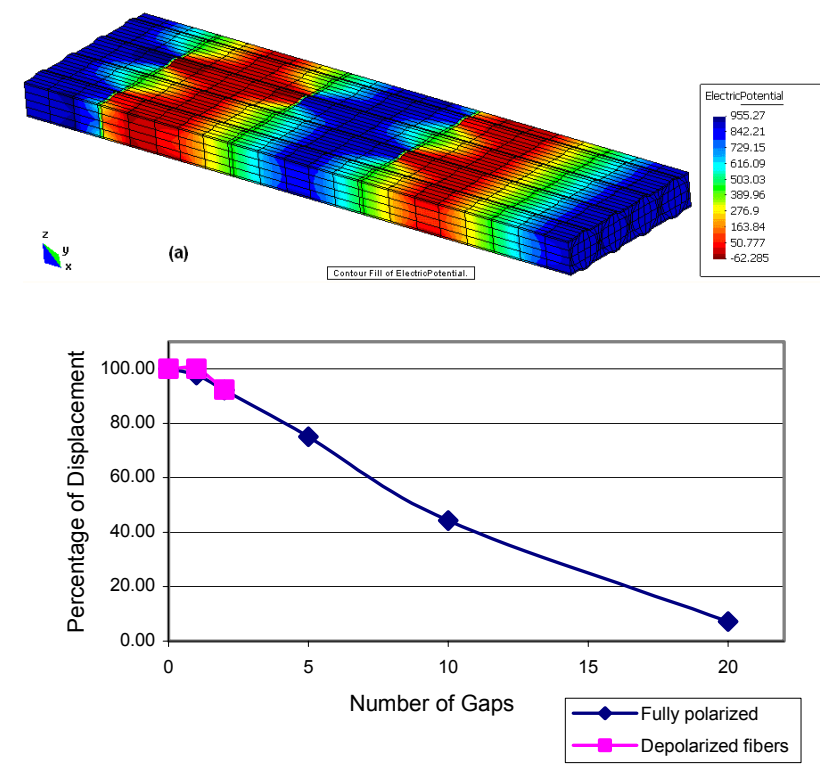

(b)

Figure 5: (a) Electric potential in the AFC under voltage applied to IDE, (b) the effect of the number of fibre gaps on the actuation performance of the AFC for systems with and without depolarized regions near gaps.

\section{Discussion and conclusions}

An experimental study of the degradation of AFC sensing properties caused by the damage produced by large strains [12] has shown that a significant loss of the sensing capacity can be produced. However, the sensing capability is almost completely restored when the applied load is reduced. Simulation results demonstrate a possible mechanism for such a reversible sensing degradation. Under a large imposed strain, broken fibres can contain wide gaps producing large depolarized regions, which result in a significant loss of the sensing function. When the load is reduced, the gaps in the fibres are closed and a fully poled state can be restored, resulting in a better sensing performance even though fibres remain mechanically broken. This simulation also demonstrates that damage in the fibres can be detrimental to both actuation and sensing performance of the $\mathrm{AFC}$; however, any significant loss of the actuation 
capability can be expected only when a large density of gaps in fibres is produced.

\section{Acknowledgement}

The authors gratefully acknowledge the financial support of Natural Sciences and Engineering Research Council of Canada (NSERC).

\section{References}

[1] Banks H.T., Smith R.C., Wang Y., Smart Material Structures - Modeling, Estimation and Control. John Wiley \& Sons, 1996.

[2] Bent A. A., "Active Fibre Composites for Structural Actuation," Ph.D. Thesis, Massachusetts Institute of Technology, January 1997.

[3] Hall S.R., Prechtl E.F. Smart Materials and Struct. 5 (1996) 26.

[4] Flinn, E.D. Aerospace America 37 (1999) 40.

[5] Odegard G.M., "Modeling of Piezoelectric Polymer Composites" National Institute of Aerospace, Hampton, Virginia. NASA/CR-2003-212681NIA Report No. 2003-10

[6] Wilkie W.K., et al., "NASA Langley Research Center Macro-Figer Composite Actuator (LaRC-MFC): Technical Overview,"

[7] Brei D., Cannon B.J., Composites Science and Tech. 64 (2004) 245.

[8] Sporn D., Schoenecker A., Mat. Res. Innovat. 2 (1999) 303.

[9] Pettermann H.E., Suresh S., Int. J. of Solids and Struct. 37 (2000) 5447.

[10] Wilkie W., High J., Bockman J., Reliability Testing of NASA Piezocomposite Actuators, U.S. Army Research Laboratory and NASA Langley Research Center, Hampton, Virginia, USA.

[11] Wickramasinghe V.K, Hagood N.W., Smart Mater. Struct. 13 (2004) 1155-1165 PII: S0964-1726 (2004) 83334-2.

[12] Melnykowycz M., et al., Performance of Integrated Active Fibre Composites in Fibre Reinforced Epoxy Laminates. to appear in Smart Materials Structures.

[13] M. Martinez, et al., Finite Element Analysis of Actuated Fibre Composites. Proceedings of CANSMART 2005, Toronto, Canada (2005) 231.

[14] Semenovskaya S., Khachaturyan A.G., J. Appl. Phys., 83 (1998) 5125.

[15] Wickramasinghe V.K, Hagood N.W., J. of Aircraft, 41 (2004) 931. 